\title{
Solute Transport With Chemical Reaction in Single- and Multi-Phase Flow in Porous Media
}

\author{
M.F. El-Amin ${ }^{1}$, \\ Amgad Salama ${ }^{2}$ and Shuyu Sun ${ }^{1}$ \\ ${ }^{1}$ King Abdullah University of Science and Technology (KAUST) \\ ${ }^{2}$ Konkuk University \\ ${ }^{1}$ Kingdom of Saudi Arabia \\ ${ }^{2}$ South Korea
}

\section{Introduction}

Transport phenomena in porous media describe the motion of fluids in media of porous structure which may be accompanied by heat/mass transfer and/or chemical reactions. While transport phenomena in fluid continua have been, to a large extent, very much comprehended, the subject matters in porous media are still under careful investigation and extensive research. Several reasons may be invoked to explain the difficulties associated with the study of transport phenomena in porous media. Probably the most obvious one is the fact that fluids move in porous media in complex, tortuous, and random passages that are even unknown a priori. Consequently, the governing laws may not be solved in any sense for the apparent difficulties in defining flow boundaries. Further complexities may be added should there exists heat transfer mechanisms associated with the flow and the interactions of heat transfer between the moving fluid and the solid matrix. Moreover, chemical reactions describe essential feature of transport in porous media. It is hardly to find transport processes in porous media without chemical reaction of some sort or another. Chemical reactions in porous media can occur naturally as a result of the interactions between the moving fluid and the surface of the solid matrix. These kinds of chemical reactions, which are usually slow, are pertinent to groundwater geochemistry, or it can be made to occur by utilizing the porous media surfaces to catalyze chemical reactions between reacting fluids. The study of these complex processes in porous media necessitate complete information about the internal structure of the porous media, which is far beyond the reach of our nowadays capacities. A fundamental question, thus, arises, in what framework do we need to cast the study of transport in porous media? In other words, do we really need to get such complete, comprehensive information about a given porous medium in order to gain useful information that could help us in our engineering applications? Do we really need to know the field variables distribution at each single point in the porous medium in order to be able to predict the evolution of this system with time, for example? Is it possible to make precise measurements within the porous media for field variables? And, even if we might be able to gain such detailed information, are we going to use them in their primitive forms for further analysis and development? The answer to these kind of questions may be that, for the sake of engineering applications, we do not need such a complete, comprehensive details, neither will we be able to obtain them nor will they 
be useful in their primitive form. In other words, field variables distribution will be randomly distributed and we would, in general, need to average them in order to gain statistically useful integral information. These ideas, in fact, enriched researchers' minds on their search for an appropriate framework to study phenomena in porous media. That is, if we need to average the pointwise field variables to get useful information, should not we might, as well, look for doing such kind of averaging on our way to investigating porous media. That is, is it possible to upscale our view to porous media such that we get smooth variables that represent integral information about the behavior of field variables not at a single point but within a volume of the porous medium surrounding this point? It turns out that researchers have appealed to this strategy several times on their way to explore the behavior of systems composed of innumerable building blocks. Thermodynamics, solid mechanics, fluid mechanics etc. are examples of sciences that adopted this approach by assuming the medium as continuum. Now, is it feasible to, also, treat phenomena occurring in porous media as continua? The answer to this question turns out to be yes as will be explained in the next section.

\section{Framework}

Salama \& van Geel (2008a) provided an interesting analogy that sheds light on the possibility to adopt the continuum approach to phenomena occurring in porous media. They stated that an observer closer to a given porous medium will be able to see details of the porous medium (at least at the surface) that an observer from far distant would, generally, ignore. To the distant observer, the medium looks smooth and homogeneous like a continuum, Fig.1. It is exactly this point of view that we seek and it remains interesting to estimate that minimum distance that our observer would have to stay to get the continuum feeling of the medium. Of course moving beyond this distance would result in no significant improvement in the continuum picture. However, moving too far without having established the continuum feeling such that the extents of the domain enter the scene implies that it would not be possible to establish the continuum picture. This analogy, in fact, gives us an idea on how to properly define upscaling to porous media and hence establish continua. That is we need an upscaling scale (volume) that is much larger than small scale heterogeneity (e.g., pore diameter) and small enough such that it does not encompass the domain boundaries.

The mathematical machinery that provide such an upscaled description to phenomena occurring in porous media includes theory of mixtures, the method of volume averaging, method of homogenization, etc. In the framework of theory of mixtures, global balance equations are written based on the assumption of the existence of macroscale field variables, which are employed in the global balance equations. A localized version of these equations may then be obtained through mathematical manipulations to get what is called the macroscopic point equations. In the frame work of the method of volume averaging, on the other hand, the microscale conservation laws adapted to fluid continua filling the interstitial space are subjected to some integral operators over representative volume which size is understood within certain set of length scale constraints. These length scale constraints were introduced for proper upscaling based on the pioneering work of several researchers including (Whitaker, Gray, Hassanizadeh, Bear, Bachmat, Quintard, Slattery, Cushman, Marle and many others). Recently, (Salama \& van Geel, 2008a) have postulated the conditions required for proper upscaling such that one may get the correct set of equations subject to the constraints pertinent to adhering to these conditions, as will be explained later. This set of macroscopic point equations, in which macroscopic field variables are defined at yet a larger scale than their microscopic counterpart, represents the governing conservation laws at the 
new macroscale. In this chapter we will be concerned mainly with the method of volume averaging.

\section{Requirements for proper averaging}

The upscaling process implies a one to one mapping between two domains one of which represents the actual porous medium and the second represents a fictitious continuous domain. Any point in the actual porous medium domain may lay on either the solid phase or the fluid phase and thus one can define a phase function which equals one if the point is in the fluid phase and is zero if it lays elsewhere. Over the actual porous medium domain, field variables are defined only over fluid phases (i.e., they only have values in the corresponding fluid-phase and zero elsewhere). These variables and/or their derivatives may not be continuous, particularly at the interfaces. The corresponding point over the fictitious medium, on the other hand, lays over a continuum where it is immaterial to talk about particular phase. Moreover, the macroscopic field variables defined over the fictitious medium are continuous over the whole domain, except possibly at the external boundaries. These macroscopic field variables represent the behavior of the fluid continuum contained within a certain volume (called representative elementary volume, REV) and are assigned to a single point in the fictitious domain. In order to succeed in establishing correctly this mapping process, extensive amount of research work have been conducted since the second half of the last century by several research groups including the pioneers mentioned earlier. On reviewing this work, (?) and recently Salama \& van Geel (2008a) proposed a set of requirements such that proper upscaling may be achieved. They require that,

1. The smoothed macroscopic variables are free from pore-related heterogeneity.

2. The smoothed macroscopic variables do not depend on the size of the averaging volume.

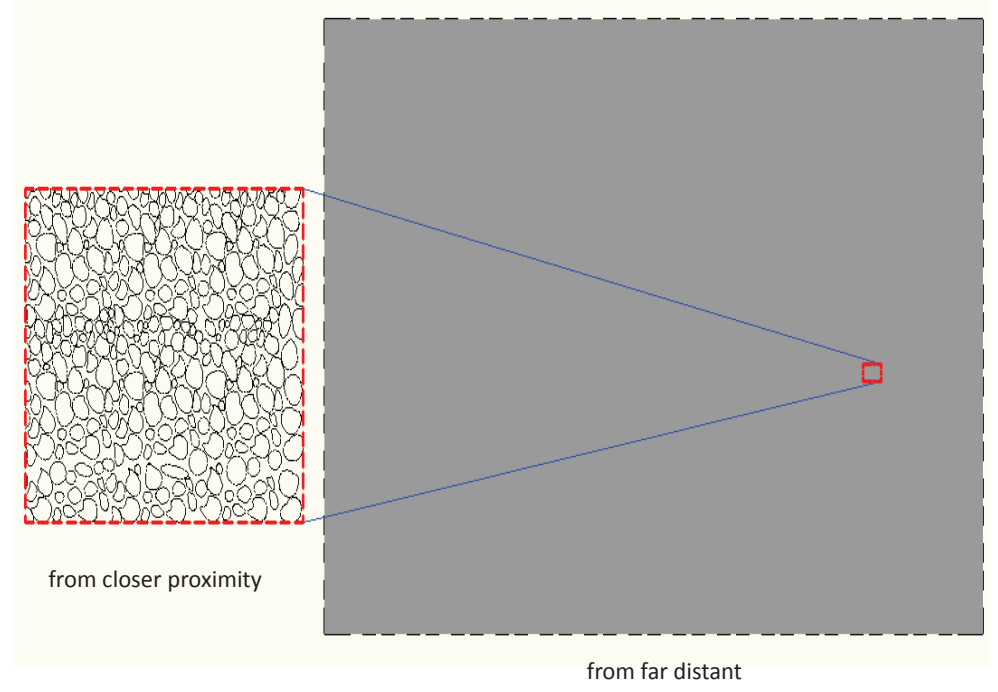

Fig. 1. View of two different observers at different proximity from a given porous medium domain. 
3. The extent of the domain under study is large enough compared with the size of the averaging volume.

4. The amount of any conservative quantity (mass, momentum, energy, etc.) within any given volume (of the size of the REV or larger) is the same if evaluated over the actual porous medium domain or the corresponding fictitious domain. And, similarly, the flux of any conservative quantity across any surface (of the size of REA or larger) is also the same in both the actual and the fictitious domains.

These requirements necessitate the followings:

- An averaging volume exists for every macroscopic field variable.

- A common range of averaging volume may be found for all the field variables.

Adhering to the first three requirements, (Whitaker, 1967) indicated that if $\ell_{\beta}$ represents a length scale pertinent to the internal microscopic structure of the porous medium (typical pore or grain diameter) and if $L$ represents a length scale associated with the extent of the domain of interest, then the length scale of the averaging volume should be such that it satisfies the following constraints
I. $\ell>>\ell_{\beta}$
II. $\ell<<L$

Adhering to the fourth requirement, on the other hand, Salama \& van Geel (2008a) were able to establish the proper averaging operator. They indicated that if we consider any conservative, intensive quantity, $\psi_{\beta}$, which may be scalar (e.g., mass of certain species per unit volume, energy per unit mass, etc.), or vector (e.g., linear momentum per unit mass). The total amount of $\psi_{\beta}$ should equal to that evaluated over the same volume in the fictitious porous medium, Fig.2, which may be evaluated as:

$$
\psi_{\text {total }}=\int_{R E V} \rho_{\beta}(\mathbf{r}, t) \psi_{\beta}(\mathbf{r}, t) \gamma_{\beta}(\mathbf{r}) d v
$$

where $\rho_{\beta}$ is the density of the $\beta$-phase, $\psi_{\beta}$ is an intensive quantity, $\gamma_{\beta}$ is the phase function, and $r$ represents the position vector spanning the REV. The time, $t$, in the argument of the indicator function, $\gamma_{\beta}$ represents the scenario of moving interfaces (e.g. immiscible multiphase system). In our case, however, the time may be omitted due to the fact that for our solid-fluid systems, the interface boundaries are assumed fixed in space. Refer to Fig.2 for a geometrical illustration.

The total amount of $\psi_{\beta}$ should equal to that evaluated over the same volume in the fictitious porous medium, Fig.3, which may be evaluated as:

$$
\psi_{\text {total }}=\int_{R E V}\left\langle\rho_{\beta}\right\rangle^{\beta}(\mathbf{r}, t)\left\langle\psi_{\beta}\right\rangle^{\beta}(\mathbf{r}, t) \epsilon_{\beta}(\mathbf{r}) d v
$$

where $\left\langle\rho_{\beta}\right\rangle^{\beta}$ and $\left\langle\psi_{\beta}\right\rangle^{\beta}$ represent the intrinsic phase average of the $\beta$-phase density and intensive quantity as described earlier in Eq. (2), and $\epsilon_{\beta}$ is the porosity function over the REV. That is,

$$
\int_{R E V} \rho_{\beta}(\mathbf{r}, t) \psi_{\beta}(\mathbf{r}, t) \gamma_{\beta}(\mathbf{r}, t) d v=\int_{R E V}\left\langle\rho_{\beta}\right\rangle^{\beta}(\mathbf{r}, t)\left\langle\psi_{\beta}\right\rangle^{\beta}(\mathbf{r}, t) \epsilon_{\beta}(\mathbf{r}) d v
$$

From the condition that the averaged quantities are assigned to the centroid of the averaging volume, (Salama \& van Geel, 2008a) postulated that, 


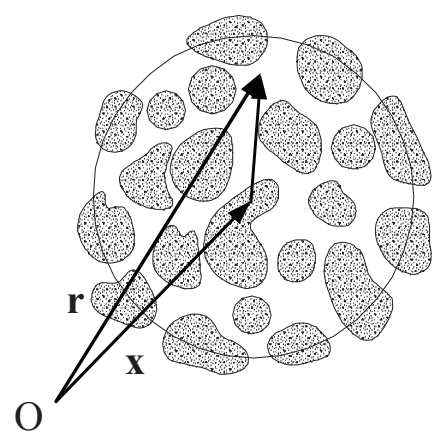

Fig. 2. An REV.

i. $\int_{R E V} \gamma_{\beta}(\mathbf{r}, t) d v=\epsilon_{\beta}(\mathbf{x}, t) v=\int_{R E V} \epsilon_{\beta}(\mathbf{r}, t) d v$

ii. $\int_{R E V} \rho_{\beta}(\mathbf{r}, t) \gamma_{\beta}(\mathbf{r}) d v=\left\langle\rho_{\beta}\right\rangle^{\beta}(\mathbf{x}, t)\left\langle\psi_{\beta}\right\rangle^{\beta}(\mathbf{x}, t) v=\int_{R E V}\left\langle\rho_{\beta}\right\rangle^{\beta}(\mathbf{r}, t) \epsilon_{\beta}(\mathbf{r}, t) d v$

iii. $\int_{R E V} \rho_{\beta}(\mathbf{r}, t) \psi_{\beta}(\mathbf{r}, t) \gamma_{\beta}(\mathbf{r}) d v=\left\langle\rho_{\beta}\right\rangle^{\beta}(\mathbf{x}, t)\left\langle\psi_{\beta}\right\rangle^{\beta}(\mathbf{x}, t) \epsilon_{\beta}(\mathbf{x}, t) v=$ $\int_{R E V}\left\langle\rho_{\beta}\right\rangle^{\beta}(\mathbf{r}, t)\left\langle\psi_{\beta}\right\rangle^{\beta}(\mathbf{r}, t) \epsilon_{\beta}(\mathbf{r}, t) d v$

where $\left\langle\rho_{\beta}\right\rangle^{\beta}(\mathbf{x}, t)\left\langle\psi_{\beta}\right\rangle^{\beta}(\mathbf{x}, t)$ and $\epsilon_{\beta}(\mathbf{x})$ are the intrinsic-phase average of $\rho_{\beta}, \psi_{\beta}$ and the porosity at the centroid of the REV, respectively (Fig. 2).

This indicates that sampling the same volume (REV) in both the actual porous medium and the fictitious one should yield the same conservative quantity. In fact this equation may be taken as the definition for the averaging processes. That is,

$$
\left\langle\psi_{\beta}\right\rangle^{\beta}(\mathbf{x}, t)=\frac{1}{\left\langle\rho_{\beta}\right\rangle^{\beta} \epsilon_{\beta} v} \int_{v} \rho_{\beta}(\mathbf{r}, t) \psi_{\beta}(\mathbf{r}, t) \gamma_{\beta}(\mathbf{r}) d v
$$

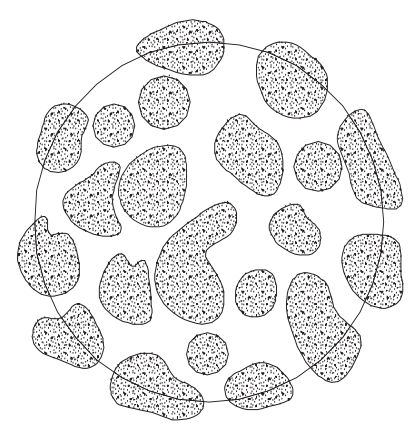

An REV over the actual porous medium.

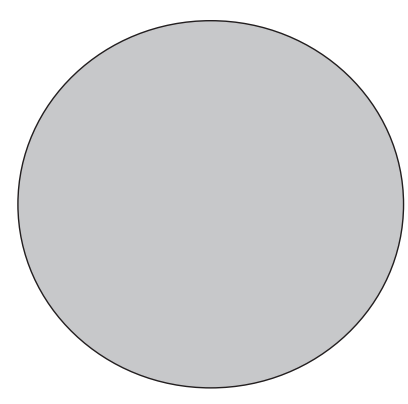

The same volume over the fictitious one.

Fig. 3. An REV over the actual porous region and an equivalent volume over the fictitious one. 
which was introduced by (Hassanizadeh \& Gray, 1979b;a; 1980) and was called the mass average of $\psi_{\beta}$. In situations where $\beta$-phase is incompressible, we have $\left\langle\rho_{\beta}\right\rangle^{\beta}=\rho_{\beta}$ and the above equation reduces to Eq. (2) and also, if $\psi_{\beta}$ represents a per unit volume quantity, for example the concentration of the $\beta$-phase, the density may be omitted. In other words the above mentioned three postulates may be used to define the averaging operators. Moreover, these postulates also suggest that: $\left\langle\left\langle\psi_{\beta}\right\rangle\right\rangle^{\beta}=\left\langle\psi_{\beta}\right\rangle$ which will show to simplify mathematical manipulation. Now for incompressible fluids Eq. (7) implies that the product $\left\langle\psi_{\beta}\right\rangle^{\beta}(\mathbf{r}, t) \epsilon_{\beta}(\mathbf{r})$ changes linearly over the REV. (Salama \& van Geel, 2008a) further indicated that, there are at least three possibilities for the product $\left\langle\psi_{\beta}\right\rangle^{\beta}(\mathbf{r}, t) \epsilon_{\beta}(\mathbf{r})$ to change linearly within the REV:

1. both $\langle\psi\rangle^{\beta}$ and $\epsilon_{\beta}$ remain constant within the REV.

2. $\langle\psi\rangle^{\beta}$ changes linearly and $\epsilon_{\beta}$ remains constant.

3. $\langle\psi\rangle^{\beta}$ remains constant and $\epsilon_{\beta}$ changes linearly.

These requirements are rather restrictive, that is, if we allow $\langle\psi\rangle^{\beta}$ and $\epsilon_{\beta}$ to vary within the averaging volume such that their product, which is apparently nonlinear, they required that the nonlinearity within the averaging volume is relatively small. They defined the criteria for this case as: if $\ell_{\epsilon}$ represents the length scale over which significant variation in porosity occur within the REV and $\ell_{\psi}$ represents that length scale over which significant deviation from the straight line variation of the intrinsic phase average of the conservative quantity $\left\langle\psi_{\beta}\right\rangle^{\beta}$ may occur, then they introduced their celebrated inequality

$$
\text { III. } \ell<<\min \left(\ell_{\epsilon}, \ell_{\psi}\right)
$$

Now adhering to these length scale constraints, it may be possible, in principle, to establish the continuum view to transport phenomena in porous media.

\section{Consequences}

As with our experience upon adopting the continuum hypothesis to the science of fluid mechanics, rather than the primitive Newtonian mechanics at the molecular scale an apparently different formulation needed to be adopted and several things arise. Probably the most obvious one is the fact that the state variables are modified. That is velocity vectors, for example, are, now, no longer associated with particles, rather they represent an integral behavior of a collection of several many particles contained within an averaging volume. The fluctuations of the actual particles velocity around continuum velocity suggest that two different mechanisms for heat and momentum transfer be hypothesized. One is associated with the transport of momentum and energy along with the continuum velocity and the other associated with the fluctuating components that appear as a surface flux in the continuum conservation laws, (Leal, 2007). We expect that these mechanisms become even more pronounced when adopting the continuum hypothesis to porous media. As an example, the dispersion of passive solute in pure liquids is very much influenced by the diffusion coefficient which is a macroscopic property of the medium. In porous media, on the other hand, dispersion becomes more pronounced and is no longer a property of the 
different fluids; it also depends on the internal geometrical structure of the porous medium. A second consequence of the continuum hypothesis is an uncertainty in the boundary conditions to be used in conjunction with the resulting macroscopic equations for motion and heat and mass transfer (Salama \& van Geel, 2008b). A third consequence is the fact that the derived macroscopic point equations contain terms at the lower scale. These terms makes the macroscopic equations unclosed. Therefore, they need to be represented in terms of macroscopic field variables though parameters that me be identified and measured.

\section{Single-phase flow modeling}

\subsection{Conservation laws}

Following the constraints introduced earlier to properly upscale equations of motion of fluid continuum to be adapted to the upscaled continuum of porous medium, researchers and scientists were able to suggest the governing laws at the new continuum. They may be written for incompressible fluids as:

Continuity

$$
\nabla \cdot\left\langle\mathbf{v}_{\beta}\right\rangle=0
$$

Momentum

$$
\begin{aligned}
& \rho_{\beta} \frac{\partial\left\langle\mathbf{v}_{\beta}\right\rangle}{\partial t}+\rho_{\beta}\left\langle\mathbf{v}_{\beta}\right\rangle^{\beta} \cdot \nabla\left\langle\mathbf{v}_{\beta}\right\rangle^{\beta}=-\nabla\left\langle p_{\beta}\right\rangle^{\beta}+\rho_{\beta} g+ \\
& \mu_{\beta} \nabla^{2}\left\langle\mathbf{v}_{\beta}\right\rangle^{\beta}-\frac{\mu_{\beta}}{K}\left\langle\mathbf{v}_{\beta}\right\rangle-\frac{\rho_{\beta} F \epsilon_{\beta}}{\sqrt{K}}\left|\left\langle\mathbf{v}_{\beta}\right\rangle\right|\left\langle\mathbf{v}_{\beta}\right\rangle
\end{aligned}
$$

Energy

$$
\sigma \frac{\partial\left\langle T_{\beta}\right\rangle^{\beta}}{\partial t}+\left\langle\mathbf{v}_{\beta}\right\rangle \cdot \nabla\left\langle T_{\beta}\right\rangle^{\beta}=k \nabla^{2}\left\langle T_{\beta}\right\rangle^{\beta} \pm Q
$$

Solute transport

$$
\epsilon \frac{\partial\left\langle c_{\beta}\right\rangle^{\beta}}{\partial t}+\left\langle\mathbf{v}_{\beta}\right\rangle \cdot \nabla\left\langle c_{\beta}\right\rangle^{\beta}=\nabla \cdot\left(D \cdot \nabla\left\langle c_{\beta}\right\rangle^{\beta}\right) \pm S
$$

where $\left\langle\mathbf{v}_{\beta}\right\rangle^{\beta}$ and $\left\langle p_{\beta}\right\rangle^{\beta}$ epresent the intrinsic average velocity and pressure, respectively and $\left\langle\mathbf{v}_{\beta}\right\rangle$ is the superficial average velocity, $\mathbf{v}=\sqrt{u^{2}+v^{2}}, \sigma=\left(\rho C_{p}\right)_{M} /\left(\rho C_{p}\right)_{f}, k=\left(k_{M} /\left(\rho C_{p}\right)_{f}\right.$, is the thermal diffusivity. From now on we will drop the averaging operator, \langle\rangle , to simplify notations. The energy equation is written assuming thermal equilibrium between the solid matrix and the moving fluid. The generic terms, $Q$ and $S$, in the energy and solute equations represent energy added or taken from the system per unit volume of the fluid per unit time and the mass of solute added or depleted per unit volume of the fluid per unit time due to some source (e.g., chemical reaction which depends on the chemistry, the surface properties of the fluid/solid interfaces, etc.). Dissolution of the solid phase, for example, adds solute to the fluid and hence $S>0$, while precipitation depletes it, i.e., $S<0$. Organic decomposition or oxidation or reduction reactions may provide both sources and sinks. Chemical reactions in porous media are usually complex that even in apparently simple processes (e.g., dissolution), sequence of steps are usually involved. This implies that the time scale of the slowest step essentially determines the time required to progress through the sequence of steps. Among 
the different internal steps, it seems that the rate-limiting step is determined by reaction kinetics. Therefore, the chemical reaction source term in the solute transport equation may be represented in terms of rate constant, $k$, which lumps several factors multiplied by the concentration, i.e.,

$$
S=k f(s)
$$

where $k$ has dimension time ${ }^{-1}$ and the form of the function $f$ may be determined experimentally, (e.g., in the form of a power law). Apparently, the above set of equations is nonlinear and hence requires, generally, numerical techniques to provide solution (finite difference, finite element, boundary element, etc.). However, in some simplified situations, one may find similarity transformations to transform the governing set of partial differential equations to a set of ordinary differential equations which greatly simplify solutions. As an example, in the following subsection we show the results of using such similarity transformations in investigating the problem of natural convection and double dispersion past a vertical flat plate immersed in a homogeneous porous medium in connection with boundary layer approximation.

\subsection{Examble: Chemical reaction in natural convection}

The present investigation describes the combined effect of chemical reaction, solutal, and thermal dispersions on non-Darcian natural convection heat and mass transfer over a vertical flat plate in a fluid saturated porous medium (El-Amin et al., 2008). It can be described as follows: A fluid saturating a porous medium is induced to flow steadily by the action of buoyancy forces originated by the combined effect of both heat and solute concentration on the density of the saturating fluid. A heated, impermeable, semi-infinite vertical wall with both temperature and concentration kept constant is immersed in the porous medium. As heat and species disperse across the fluid, its density changes in space and time and the fluid is induced to flow in the upward direction adjacent to the vertical plate. Steady state is reached when both temperature and concentration profiles no longer change with time. In this study, the inclusion of an n-order chemical reaction is considered in the solute transport equation. On the other hand, the non-Darcy (Forchheimer) term is assumed in the flow equations. This term accounts for the non-linear effect of pore resistance and was first introduced by Forchheimer. It incorporates an additional empirical (dimensionless) constant, which is a property of the solid matrix, (Herwig \& Koch, 1991). Thermal and mass diffusivities are defined in terms of the molecular thermal and solutal diffusivities, respectively. The Darcy and non-Darcy flow, temperature and concentration fields in porous media are observed to be governed by complex interactions among the diffusion and convection mechanisms as will be discussed later. It is assumed that the medium is isotropic with neither radiative heat transfer nor viscous dissipation effects. Moreover, thermal local equilibrium is also assumed. Physical model and coordinate system is shown in Fig.4.

The $x$-axis is taken along the plate and the $y$-axis is normal to it. The wall is maintained at constant temperature and concentration, $T_{w}$ and $C_{w}$, respectively. The governing equations for the steady state scenario [as given by (Mulolani \& Rahman, 2000; El-Amin, 2004) may be presented as:

Continuity:

$$
\frac{\partial u}{\partial x}+\frac{\partial v}{\partial y}=0
$$




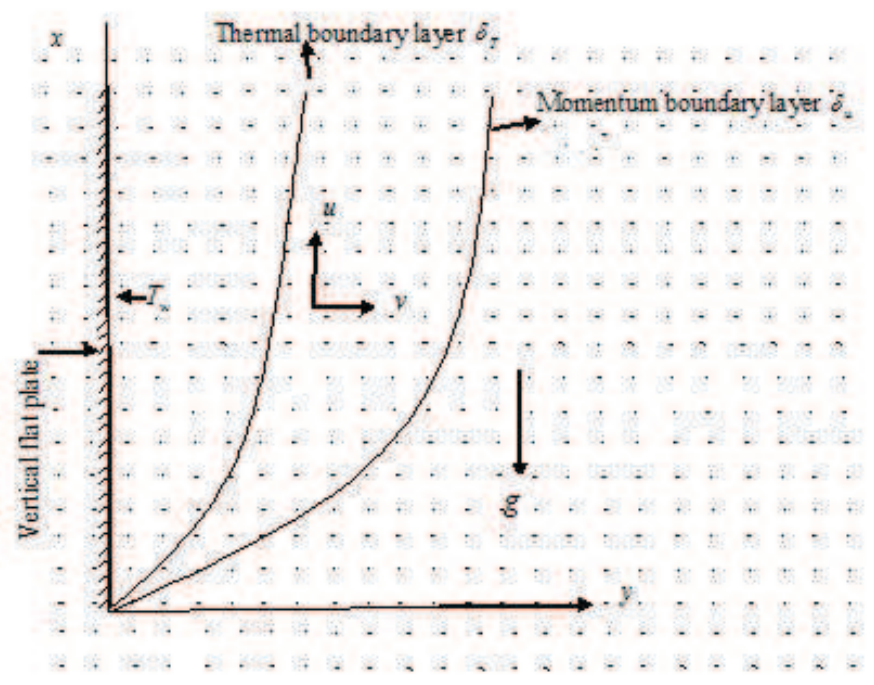

Fig. 4. Physical model and coordinate system.

Momentum:

$$
\begin{gathered}
u \frac{c \sqrt{K}}{v} u|\mathbf{v}|=-\frac{K}{\mu}\left(\frac{\partial p}{\partial x}+\rho g\right) \\
v \frac{c \sqrt{K}}{v} v|\mathbf{v}|=-\frac{K}{\mu}\left(\frac{\partial p}{\partial y}\right)
\end{gathered}
$$

Energy:

$$
u \frac{\partial T}{\partial x}+v \frac{\partial T}{\partial y}=\frac{\partial}{\partial x}\left(\alpha_{x} \frac{\partial T}{\partial x}\right)+\frac{\partial}{\partial y}\left(\alpha_{y} \frac{\partial T}{\partial y}\right)
$$

Solute transport:

$$
u \frac{\partial C}{\partial x}+v \frac{\partial C}{\partial y}=\frac{\partial}{\partial x}\left(D_{x} \frac{\partial C}{\partial x}\right)+\frac{\partial}{\partial y}\left(D_{y} \frac{\partial C}{\partial y}\right)-K_{0}\left(C-C_{\infty}\right)^{n}
$$

Density

$$
\rho=\rho_{\infty}\left[1-\beta^{*}\left(T-T_{\infty}\right)-\beta^{* *}\left(C-C_{\infty}\right)\right]
$$

Along with the boundary conditions:

$$
\begin{aligned}
& y=0: v=0, T_{w}=\text { const., } C_{w}=\text { const.; } \\
& y \rightarrow \infty: u=0, T \rightarrow T_{\infty}, C \rightarrow C_{\infty}
\end{aligned}
$$

where $\beta^{*}$ is the thermal expansion coefficient $\beta^{* *}$ is the solutal expansion coefficient. It should be noted that $u$ and $v$ refers to components of the volume averaged (superficial) velocity of the fluid. The chemical reaction effect is acted by the last term in the right hand side of Eq. (14), where, the power $n$ is the order of reaction and $K_{0}$ is the chemical reaction constant. It is assumed that the normal component of the velocity near the boundary is small compared 
with the other component of the velocity and the derivatives of any quantity in the normal direction are large compared with derivatives of the quantity in direction of the wall. Under these assumptions, Eq. (10) remains the same, while Eqs. (11)- (15) become:

$$
\begin{gathered}
u+\frac{c \sqrt{K}}{v} u^{2}=-\frac{K}{\mu}\left(\frac{\partial p}{\partial x}+\rho g\right) \\
\frac{\partial p}{\partial y}=0 \\
u \frac{\partial T}{\partial x}+v \frac{\partial T}{\partial y}=\frac{\partial}{\partial y}\left(\alpha_{y} \frac{\partial T}{\partial y}\right) \\
u \frac{\partial C}{\partial x}+v \frac{\partial C}{\partial y}=\frac{\partial}{\partial y}\left(D_{y} \frac{\partial C}{\partial y}\right)-K_{0}\left(C-C_{\infty}\right)^{n}
\end{gathered}
$$

Following (Telles \& V.Trevisan, 1993), the quantities of $\alpha_{y}$ and $D_{y}$ are variables defined as $\alpha_{y}=\alpha+\gamma d|v|$ and $D_{y}=D+\zeta d|v|$ where, $\alpha$ and $D$ are the molecular thermal and solutal diffusivities, respectively, whereas $\gamma d|v|$ and $\zeta d|v|$ represent dispersion thermal and solutal diffusivities, respectively. This model for thermal dispersion has been used extensively (e.g., (Cheng, 1981; Plumb, 1983; Hong \& Tien, 1987; Lai \& Kulacki, 1989; Murthy \& Singh, 1997) in studies of non-Darcy convective heat transfer in porous media. Invoking the Boussinesq approximations, and defining the velocity components $u$ and $v$ in terms of stream function $\psi$ as: $u=\partial \psi / \partial y$ and $v=-\partial \psi / \partial x$, the pressure term may be eliminated between Eqs. (17) and (18) and one obtains:

$$
\begin{gathered}
\frac{\partial^{2} \psi}{\partial y^{2}}+\frac{c \sqrt{K}}{v} \frac{\partial}{\partial y}\left(\frac{\partial \psi}{\partial y}\right)^{2}=\left(\frac{K g \beta^{*}}{\mu} \frac{\partial T}{\partial y}+\frac{K g \beta^{* *}}{\mu} \frac{\partial C}{\partial y}\right) \rho_{\infty} \\
\frac{\partial \psi}{\partial y} \frac{\partial T}{\partial x}-\frac{\partial \psi}{\partial x} \frac{\partial T}{\partial y}=\frac{\partial}{\partial y}\left[\left(\alpha+\gamma d \frac{\partial \psi}{\partial y}\right) \frac{\partial T}{\partial y}\right] \\
\frac{\partial \psi}{\partial y} \frac{\partial C}{\partial x}-\frac{\partial \psi}{\partial x} \frac{\partial C}{\partial y}=\frac{\partial}{\partial y}\left[\left(D+\zeta d \frac{\partial \psi}{\partial y}\right) \frac{\partial C}{\partial y}\right]-K_{0}\left(C-C_{\infty}\right)^{n}
\end{gathered}
$$

Introducing the similarity variable and similarity profiles (El-Amin, 2004):

$$
\eta=R a_{x}^{1 / 2} \frac{y}{x}, f(\eta)=\frac{\psi}{\alpha R a_{x}^{1 / 2}}, \theta(\eta)=\frac{T-T_{\infty}}{T_{w}-T_{\infty}}, \phi(\eta)=\frac{C-C_{\infty}}{C_{w}-C_{\infty}}
$$

The problem statement is reduced to:

$$
\begin{gathered}
f^{\prime \prime}+2 F_{0} R a_{d} f^{\prime} f^{\prime \prime}=\theta^{\prime}+N \phi^{\prime} \\
\theta^{\prime \prime}+\frac{1}{2} f \theta^{\prime}+\gamma R a_{d}\left(f^{\prime} \theta^{\prime \prime}+f^{\prime \prime} \theta^{\prime}\right)=0 \\
\phi^{\prime \prime}+\frac{1}{2} L e f \phi^{\prime}+\zeta L e R a_{d}\left(f^{\prime} \phi^{\prime \prime}+f^{\prime \prime} \phi^{\prime}\right)-S c \lambda \frac{G c}{R e_{x}^{2}} \phi^{n=0}
\end{gathered}
$$

As mentioned in (El-Amin, 2004), the parameter $F_{0}=c \sqrt{K} \alpha / v d$ collects a set of parameters that depend on the structure of the porous medium and the thermo physical properties of the fluid saturating it, $R a_{d}=K g \beta^{*}\left(T_{w}-T_{\infty}\right) d / \alpha v$ is the modified, pore-diameter-dependent 
Rayleigh number, and $N=\beta^{* *}\left(C_{w}-C_{\infty}\right) / \beta^{*} v$ is the buoyancy ratio parameter. With analogy to (Mulolani \& Rahman, 2000; Aissa \& Mohammadein, 2006), we define Gc to be the modified Grashof number, $R e_{x}$ is local Reynolds number, $S c$ and $\lambda$ are Schmidt number and non-dimensional chemical reaction parameter defined as $G_{c}=\beta^{* *} g\left(C_{w}-C_{\infty}\right)^{2} x^{3} / v^{2}, R e_{x}=$ $u_{r} x / \nu, S c=v / D$ and $\lambda=K_{0} \alpha d\left(C_{w}-C_{\infty}\right)^{n-3} / K g \beta^{* *}$, where the diffusivity ratio Le (Lewis number) is the ratio of Schmidt number and Prandtl number, and $u_{r}=\sqrt{g \beta^{*} d\left(T_{w}-T_{\infty}\right)}$ is the reference velocity as defined by (Elbashbeshy, 1997).

Eq. (27) can be rewritten in the following form:

$$
\phi^{\prime \prime}+\frac{1}{2} L e f \phi^{\prime}+\zeta L e R a_{d}\left(f^{\prime} \phi^{\prime \prime}+f^{\prime \prime} \phi^{\prime}\right)-\chi \phi^{n}=0
$$

With analogy to (Prasad et al., 2003; Aissa \& Mohammadein, 2006), the non-dimensional chemical reaction parameter $\chi$ is defined as $\chi=S c \lambda G c / R e_{x}^{2}$. The boundary conditions then become:

$$
f(0)=0, \theta(0)=\phi(0)=1, f^{\prime}(\infty)=\theta(\infty)=\phi(\infty)=0
$$

It is noteworthy to state that $F_{0}=0$ corresponds to the Darcian free convection regime, $\gamma=0$ represents the case where the thermal dispersion effect is neglected and $\zeta=0$ represents the case where the solutal dispersion effect is neglected. In Eq. (16), $N>0$ indicates the aiding buoyancy and $N<0$ indicates the opposing buoyancy. On the other hand, from the definition of the stream function, the velocity components become $u=\left(\alpha R a_{x} / x\right) f^{\prime}$ and $v=-\left(\alpha R a_{x}^{1 / 2} / 2 x\right)\left[f-\eta f^{\prime}\right]$. The local heat transfer rate which is one of the primary interest of the study is given by $q_{w}=-\left.k_{e}(\partial T / \partial y)\right|_{y=0}$, where, $k_{e}=k+k_{d}$ is the effective thermal conductivity of the porous medium which is the sum of the molecular thermal conductivity $k$ and the dispersion thermal conductivity $k_{d}$. The local Nusselt number $N u_{x}$ is defined as $N u_{x}=q_{w} x /\left(T_{w}-T_{\infty}\right) k_{e}$. Now the set of primary variables which describes the problem may be replaced with another set of dimensionless variables. This include: a dimension less variable that is related to the process of heat transfer in the given system which may be expressed as $N u_{x} / \sqrt{R a_{x}}=-\left[1+\gamma R a_{d} F^{\prime}(0)\right] \theta^{\prime}(0)$. Also, the local mass flux at the vertical wall that is given by $j_{w}=-\left.D_{y}(\partial C / \partial y)\right|_{y=0}$ defines another dimensionless variable that is the local Sherwood number is given by, $S h_{x}=j_{w} x /\left(C_{w}-C_{\infty}\right) D$. This, analogously, may also define another dimensionless variable as $S h_{x} / \sqrt{R a_{x}}=-\left[1+\zeta R a_{d} F^{\prime}(0)\right] \phi^{\prime}(0)$.

The details of the effects of all these parameters are presented in (El-Amin et al., 2008). We, however, highlight the role of the chemical reaction on this system. The effect of chemical reaction parameter $\chi$ on the concentration as a function of the boundary layer thickness $\eta$ and with respect to the following parameters: $L e=0.5, F_{0}=0.3, R a_{d}=0.7, \gamma=\zeta=0.0$, $N=-0.1$ are plotted in Fig.5. This figure indicates that increasing the chemical reaction parameter decreases the concentration distributions, for this particular system. That is, chemical reaction in this system results in the consumption of the chemical of interest and hence results in concentration profile to decrease. Moreover, this particular system also shows the increase in chemical reaction parameter $\chi$ to enhance mass transfer rates (defined in terms of Sherwood number) as shown in Fig.6. It is worth mentioning that the effects of chemical reaction on velocity and temperature profiles as well as heat transfer rate may be negligible. Figs. 7 and 8 illustrate, respectively, the effect of Lewis number Le on Nusselt number and Sherwood number for various with the following parameters set as $\chi=0.02$, $R a_{d}=0.7, F_{0}=0.3, N=-0.1, \gamma=0.0$. The parameter $\zeta$ seems to reduce the heat transfer rates especially with higher Le number as shown in Fig. 7. In the case of mass transfer rates 


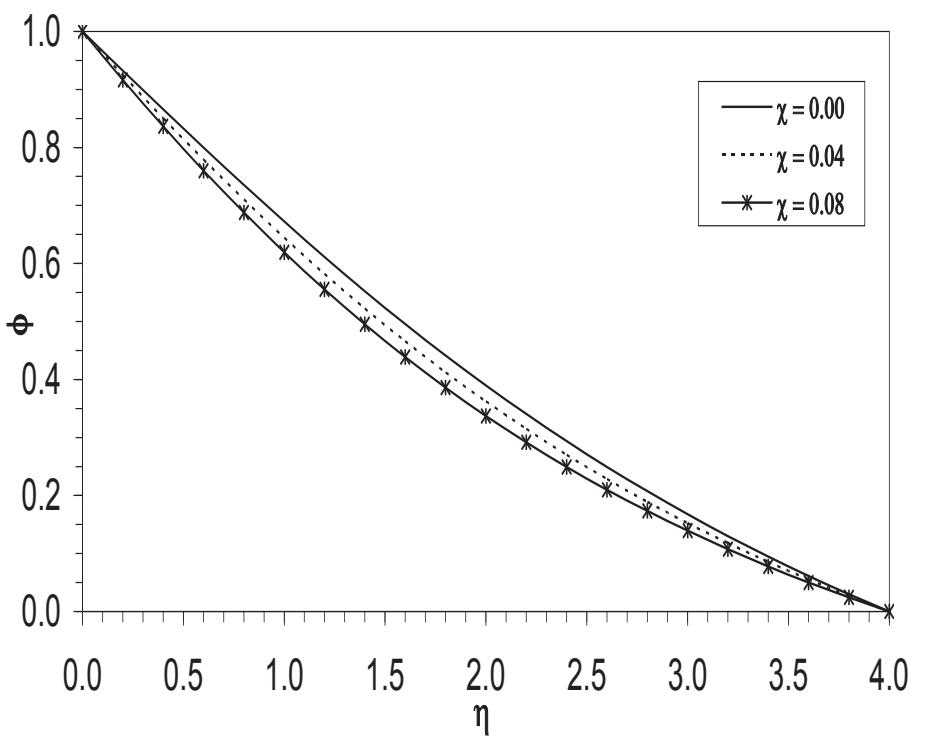

Fig. 5. Variation of dimensionless concentration with similarity space variable $\eta$ for different $\chi\left(L e=0.5, F_{0}=0.3, R a_{d}=0.7, \gamma=\zeta=0.0, N=-0.1\right)$.

(defined in terms of Sherwood number), Fig. 8 illustrates that the parameter $\zeta$ enhances the

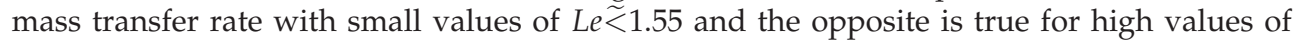
Le $>1.55$. This may be explained as follows: for small values of Le number, which indicates

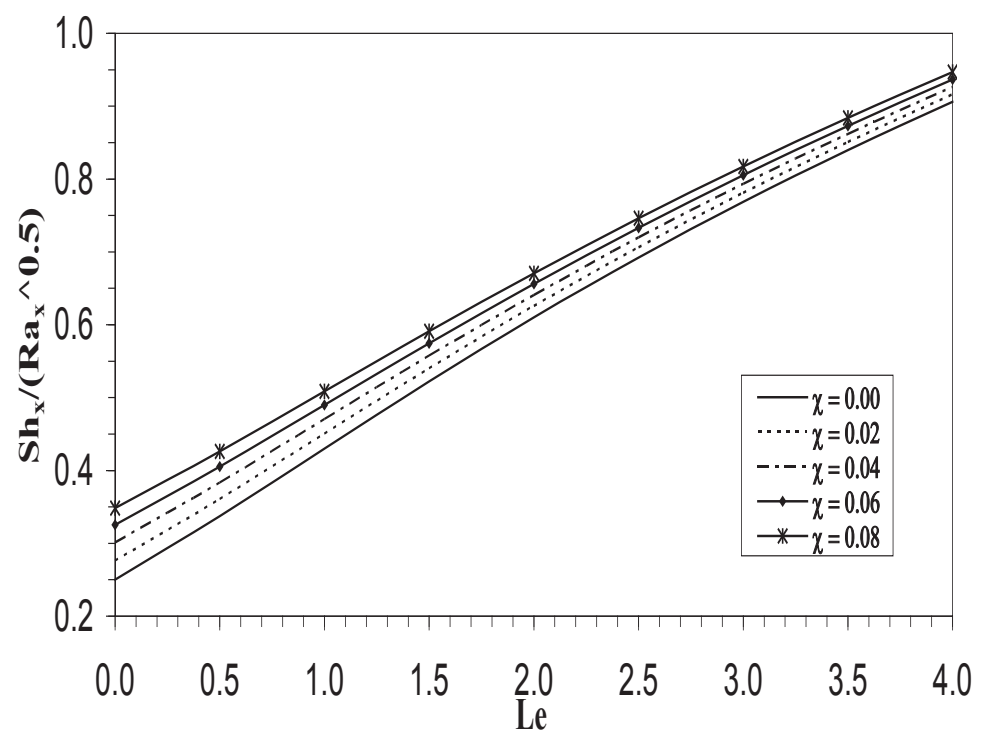

Fig. 6. Effect of Lewis number on Sherwood number for various $\chi\left(F_{0}=0.3, R a_{d}=0.7\right.$, $\gamma=\zeta=0.0, N=-0.1)$. 


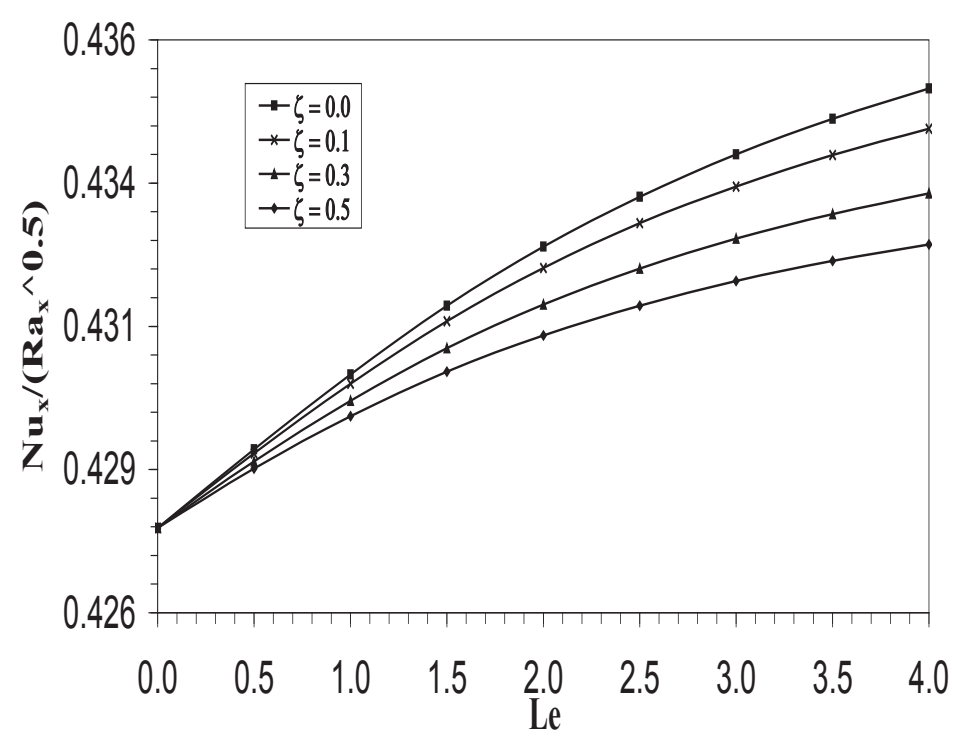

Fig. 7. Variation of Nusselt number with Lewis number for various $\zeta\left(\chi=0.02, F_{0}=0.3\right.$, $\left.R a_{d}=0.7, \gamma=0.0, N=-0.1\right)$.

that mass dispersion outweighs heat dispersion, the increase in the parameter $\zeta$ causes mass dispersion mechanism to be higher and since the concentration at the wall is kept constant this increases concentration gradient near the wall and hence increases Sherwood number. As

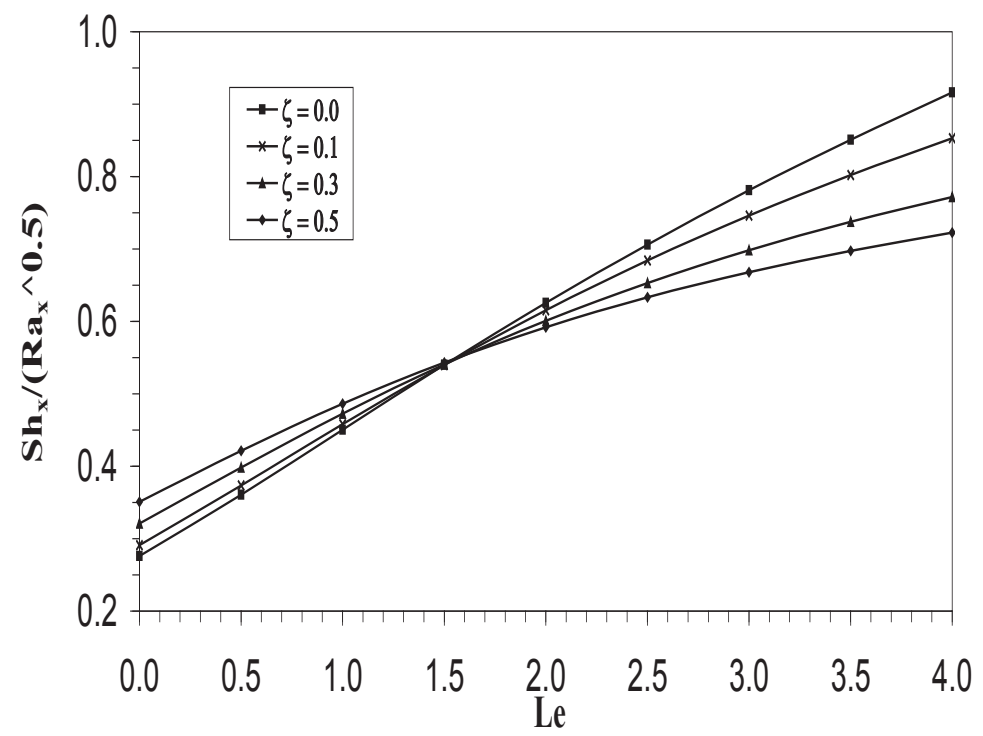

Fig. 8. Effect of Lewis number on Sherwood number for various $\zeta\left(\chi=0.02, F_{0}=0.3\right.$, $\left.R a_{d}=0.7, \gamma=0.0, N=-0.1\right)$. 


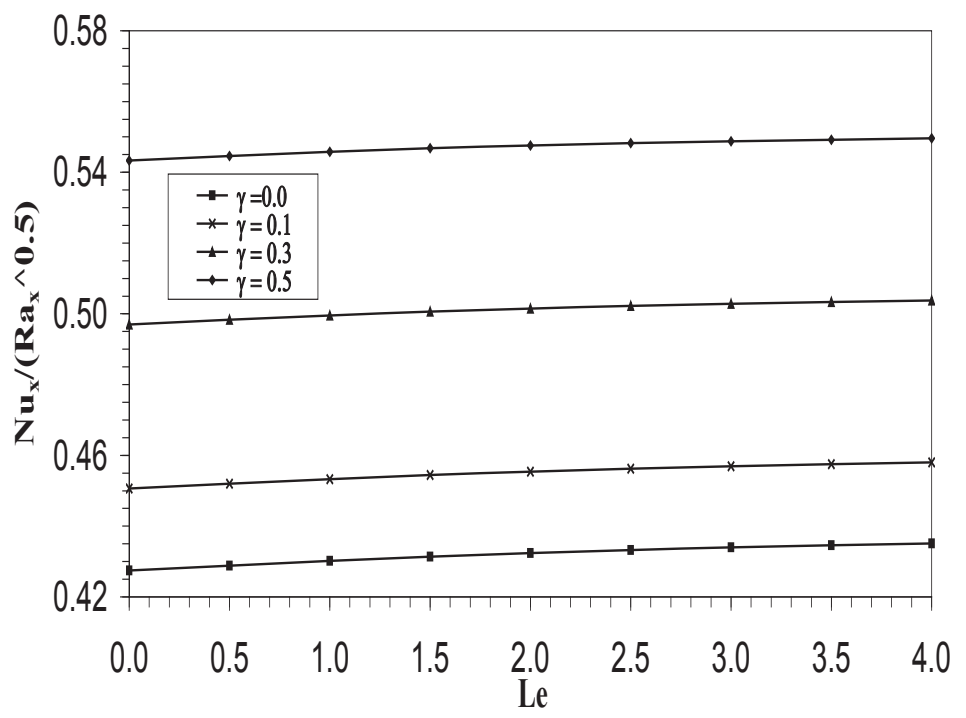

Fig. 9. Variation of Nusselt number with Lewis number for various $\gamma\left(\chi=0.02, F_{0}=0.3\right.$, $\left.R a_{d}=0.7, \zeta=0.0, N=-0.1\right)$.

$L e$ increases $(L e>1)$, heat dispersion outweighs mass dispersion and with the increase in $\zeta$ concentration gradient near the wall becomes smaller and this results in decreasing Sherwood number. Fig. 9 indicates that the increase in thermal dispersion parameter enhances the heat transfer rates.

\section{Multi-phase flow modeling}

Multi-phase systems in porous media are ubiquitous either naturally in connection with, for example, vadose zone hydrology, which involves the complex interaction between three phases (air, groundwater and soil) and also in many industrial applications such as enhanced oil recovery (e.g., chemical flooding and $\mathrm{CO}_{2}$ injection), Nuclear waste disposal, transport of groundwater contaminated with hydrocarbon (NAPL, DNAPL), etc. Modeling of Multi-phase flows in porous media is, obviously, more difficult than in single-phase systems. Here we have to account for the complex interfacial interactions between phases as well as the time dependent deformation they undergo. Modeling of compositional flows in porous media is, therefore, necessary to understand a number of problems related to the environment (e.g., $\mathrm{CO}_{2}$ sequestration) and industry (e.g., enhanced oil recovery). For example, $\mathrm{CO}_{2}$ injection in hydrocarbon reservoirs has a double benefit, on the one side it is a profitable method due to issues related to global warming, and on the other hand it represents an effective mechanism in hydrocarbon recovery. Modeling of these processes is difficult because the several mechanisms involved. For example, this injection methodology associates, in addition to species transfer between phases, some substantial changes in density and viscosity of the phases. The number of phases and compositions of each phase depend on the thermodynamic conditions and the concentration of each species. Also, multi-phase compositional flows have varies applications in different areas such as nuclear reactor safety analysis (Dhir, 1994), 
high-level radioactive waste repositories (Doughty \& Pruess, 1988), drying of porous solids and soils (Whitaker, 1977), porous heat pipes (Udell, 1985), geothermal energy production (Cheng, 1978), etc. The mathematical formulation of the transport phenomena are governed by conservation principles for each phase separately and by appropriate interfacial conditions between various phases. Firstly we give the general governing equations of multi-phase, multicomponent transport in porous media. Then, we provide them in details with analysis for two- and three-phase flows. The incompressible multi-phase compositional flow of immiscible fluids are described by the mass conservation in a phase (continuity equation), momentum conservation in a phase (generalized Darcy's equation) and mass conservation of component in phase (spices transport equation). The transport of $N$-components of multi-phase flow in porous media are described by the molar balance equations. Mass conservation in phase $\alpha$ :

$$
\frac{\partial\left(\phi \rho_{\alpha} S_{\alpha}\right)}{\partial t}=-\nabla \cdot\left(\rho_{\alpha} u_{\alpha}\right)+q_{\alpha}
$$

Momentum conservation in phase $\alpha$ :

$$
u_{\alpha}=-\frac{K k_{r \alpha}}{\mu_{\alpha}}\left(\nabla p_{\alpha}+\rho_{\alpha} g \nabla z\right)
$$

Energy conservation in phase $\alpha$ :

$$
\frac{\partial}{\partial t}\left(\epsilon \rho_{\alpha} S_{\alpha} h_{\alpha}\right)+\nabla \cdot\left(\rho_{\alpha} u_{\alpha} h_{\alpha}\right)=\nabla \cdot\left(\epsilon S_{\alpha} k_{\alpha} \nabla T\right)+\bar{q}_{\alpha}
$$

Mass conservation of component $i$ in phase $\alpha$ :

$$
\frac{\partial\left(\phi c z_{i}\right)}{\partial t}+\nabla \cdot \sum_{\alpha} c_{\alpha} x_{\alpha i} u_{\alpha}=\nabla \cdot\left(\phi D_{\alpha}^{i} \nabla\left(c z_{i}\right)\right)+F_{i}, \quad i=1, \cdots, N
$$

where the index $\alpha$ denotes to the phase. $S, p, q, u, k_{r}, \rho$ and $\mu$ are the phase saturation, pressure, mass flow rate, Darcy velocity, relative permeability, density and viscosity, respectively. $c$ is the overall molar density; $z_{i}$ is the total mole fraction of $i^{\text {th }}$ component; $c_{\alpha}$ is the phase molar densities; $x_{\alpha i}$ is the phase molar fractions; and $F_{i}$ is the source/sink term of the $i^{\text {th }}$ component which can be considered as the phase change at the interface between the phase $\alpha$ and other phases; and/or the rate of interface transfer of the component $i$ caused by chemical reaction (chemical non-equilibrium). $D_{\alpha}^{i}$ is a macroscopic second-order tensor incorporating diffusive and dispersive effects. The local thermal equilibrium among phases has been assumed, $\left(T_{\alpha}=T, \forall \alpha\right)$, and $k_{\alpha}$ and $\bar{q}_{\alpha}$ represent the effective thermal conductivity of the phase $\alpha$ and the interphase heat transfer rate associated with phase $\alpha$, respectively. Hence, $\sum_{\alpha} \bar{q}_{\alpha}=q, q$ is an external volumetric heat source/sink (Starikovicius, 2003). The phase enthalpy $k_{\alpha}$ is related to the temperature $T$ by, $h_{\alpha}=\int_{0}^{T} c_{p \alpha} d T+h_{\alpha}^{0}$. The saturation $S_{\alpha}$ of the phases are constrained by, $c_{p \alpha}$ and $h_{\alpha}^{0}$ are the specific heat and the reference enthalpy oh phase $\alpha$, respectively.

$$
\sum_{\alpha} S_{\alpha}=1
$$

One may defined the phase saturation as the fraction of the void volume of a porous medium filled by this fluid phase. The mass flow rate $q_{\alpha}$, describe sources or sinks and can be defined by the following relation (Chen, 2007), 


$$
\begin{gathered}
q=\sum_{j} \rho^{j} q^{j} \delta\left(x-x^{j}\right) \\
q=-\sum_{j} \rho^{j} q^{j} \delta\left(x-x^{j}\right)
\end{gathered}
$$

The index $j$ represents the points of sources or sinks. Eq. (35) represents sources and $q^{j}$ represents volume of the fluid (with density $\rho^{j}$ ) injected per unit time at the points locations $x^{j}$, while, Eq. (36) represents sinks and $q^{j}$ represents volume of the fluid produced per unit time at $x^{j}$.

On the other hand, the molar density of wetting and nonwetting phases is given by,

$$
c_{\alpha}=\sum_{i=1}^{N} c_{\alpha i}
$$

where $c_{\alpha i}$ is the molar densities of the component $i$ in the phase $\alpha$. Therefore, the mole fraction of the component $i$ in the respective phase is given as,

$$
x_{\alpha i}=\frac{c_{\alpha i}}{c_{\alpha}}, \quad i=1, \cdots, N
$$

The mole fraction balance implies that,

$$
\sum_{i=1}^{N} x_{\alpha i}=1
$$

Also, for the total mole fraction of $i^{\text {th }}$ component,

$$
\sum_{i=1}^{N} z_{i}=1
$$

Alternatively, Eq. (32) can be rewritten in the following form,

$$
\frac{\partial}{\partial t}\left(\phi \sum_{\alpha} c_{\alpha} x_{\alpha i} S_{\alpha}\right)+\nabla \cdot \sum_{\alpha} c_{\alpha} x_{\alpha i} u_{\alpha}=\nabla \cdot\left(\phi c_{\alpha} S_{\alpha} D_{\alpha}^{i} \nabla x_{\alpha i}\right)+F_{i}, \quad i=1, \cdots, N
$$

$F_{i}$ may be written as,

$$
F_{i}=\sum_{\alpha} x_{\alpha i} q_{\alpha}, \quad i=1, \cdots, N
$$

where $q_{\alpha}$ is the phase flow rate given by Eqs. (35), (36). From Eqs. (32) and (41), one may deduce,

$$
c z_{i}=\sum_{\alpha} c_{\alpha} x_{\alpha i} S_{\alpha}=\sum_{\alpha} c_{\alpha i} S_{\alpha}, \quad i=1, \cdots, N
$$

If one uses the total mass variable X of the system (Nolen 1973; Young and Stephenson 1983),

$$
X=\sum_{\alpha} c_{\alpha} S_{\alpha}
$$

Therefore, 


$$
1=\sum_{\alpha} \frac{c_{\alpha} S_{\alpha}}{X}=\sum_{\alpha} C_{\alpha}
$$

where $C_{\alpha}$ is the mass fraction phase $\alpha$, respectively.

The quantity,

$$
k_{\alpha}=K k_{r \alpha}
$$

is known as effective permeability of the phase $\alpha$. The relative permeability of a phase is a dimensionless measure of the effective permeability of that phase. It is the ratio of the effective permeability of that phase to the absolute permeability. Also, it is interesting to define the quantities $m_{\alpha}$ which is known as mobility ratios of phases $\alpha$, respectively are given by,

$$
m_{\alpha}=\frac{k_{\alpha}}{\mu_{\alpha}}
$$

The capillary pressure is the the difference between the pressures for two adjacent phases $\alpha_{1}$ and $\alpha_{2}$, given as,

$$
p_{c \alpha_{1} \alpha_{2}}=p_{\alpha_{1}}-p_{\alpha_{2}}
$$

The capillary pressure function is dependent on the pore geometry, fluid physical properties and phase saturations. The two phase capillary pressure can be expressed by Leverett dimensionless function $J(S)$, which is a function of the normalized saturation $S$,

$$
p_{c}=\gamma\left(\frac{\phi}{K}\right)^{\frac{1}{2}} J(S)
$$

The $J(S)$ function typically lies between two limiting (drainage and imbibition) curves which can be obtained experimentally.

\subsection{Two-phase compositional flow}

The governing equations of two-phase compositional flow of immiscible fluids are given by, Mass conservation in phase $\alpha$ :

$$
\frac{\partial\left(\phi \rho_{\alpha} S_{\alpha}\right)}{\partial t}=-\nabla \cdot\left(\rho_{\alpha} u_{\alpha}\right)+q_{\alpha} \quad \alpha=w, n
$$

Momentum conservation in phase $\alpha$ :

$$
u_{\alpha}=-\frac{K k_{r \alpha}}{\mu_{\alpha}}\left(\nabla p_{\alpha}+\rho_{\alpha} g \nabla z\right) \quad \alpha=w, n
$$

Mass conservation of component $i$ in phase $\alpha$ :

$$
\frac{\partial\left(\phi c z_{i}\right)}{\partial t}+\nabla \cdot\left(c_{w} x_{w i} u_{w}+c_{n} x_{n i} u_{n}\right)=F_{i}, \quad i=1, \cdots, N
$$

where the index $\alpha$ denotes to the wetting $(w)$ and non-wetting $(n)$, respectively. $S, p, q, u, k_{r}, \rho$ and $\mu$ are the phase saturation, pressure, mass flow rate, Darcy velocity, relative permeability, density and viscosity, respectively. $c$ is the overall molar density; $z_{i}$ is the total mole fraction of $i^{t h}$ component; $c_{w}, c_{n}$ are the wetting- and nonwetting-phase molar densities; $x_{w i}, x_{n i}$ are the wetting- and nonwetting-phase molar fractions; and $F_{i}$ is the source/sink term of the $i^{\text {th }}$ component. The saturation $S_{\alpha}$ of the phases are constrained by, 


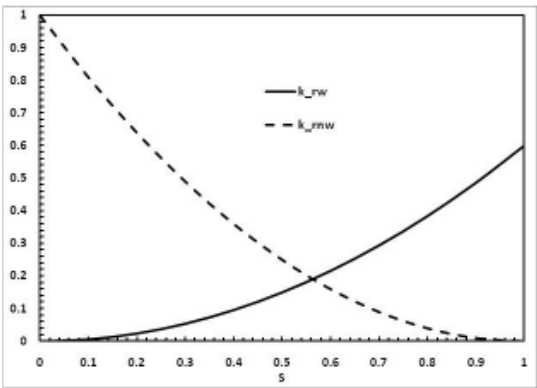

(a) Corey approximation

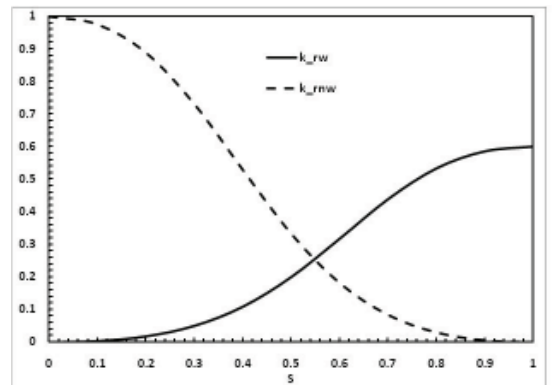

(b) LET approximation

Fig. 10. Relative permeabilities.

$$
S_{w}+S_{n}=1
$$

The normalized wetting phase saturation $S$ is given by,

$$
S=\frac{S_{w}-S_{0 w}}{1-S_{n r}-S_{0 w}} \quad 0 \leq S \leq 1
$$

where $S_{0 w}$ is the irreducible (minimal) wetting phase saturation and $S_{n r}$ is the residual (minimal) non-wetting phase saturation. The expression of relation between the relative permeabilities and the normalized wetting phase saturation $S$, given as,

$$
\begin{gathered}
k_{r w}=k_{r w}^{0} S^{a} \\
k_{r n}=k_{r n}^{0}(1-S)^{b}
\end{gathered}
$$

The empirical parameters $a$ and $b$ can be obtained from measured data either by optimizing to analytical interpretation of measured data, or by optimizing using a core flow numerical simulator to match the experiment. $k_{r w}^{0}=k_{r w}(S=1)$ is the endpoint relative permeability to water, and $k_{r n}^{0}=k_{r n}(S=0)$ is the endpoint relative permeability to the non-wetting phase.

For example, for the Corey power-law correlation, $a=b=2, k_{r n}^{0}=1, k_{r w}^{0}=0.6$, for water-oil system see Fig.10a. Another example of relative permeabilities correlations is LET model which is more accurate than Corey model. The LET-type approximation is described by three empirical parameters $\mathrm{L}, \mathrm{E}$ and $\mathrm{T}$. The relative permeability correlation for water-oil system has the form,

$$
k_{r w}=\frac{k_{r w}^{0} S^{L_{w}}}{S^{L_{w}}+E_{w}(1-S)^{T_{w}}}
$$

and

$$
k_{r n}=\frac{(1-S)^{L_{n}}}{(1-S)^{L_{n}}+E_{n} S^{T_{n}}}
$$

The parameter $E$ describes the position of the slope (or the elevation) of the curve. Fig. 10b shows LET relative permeabilities with $L=E=T=2$ and $k_{r w}^{0}=0.6$ for water-oil system. 


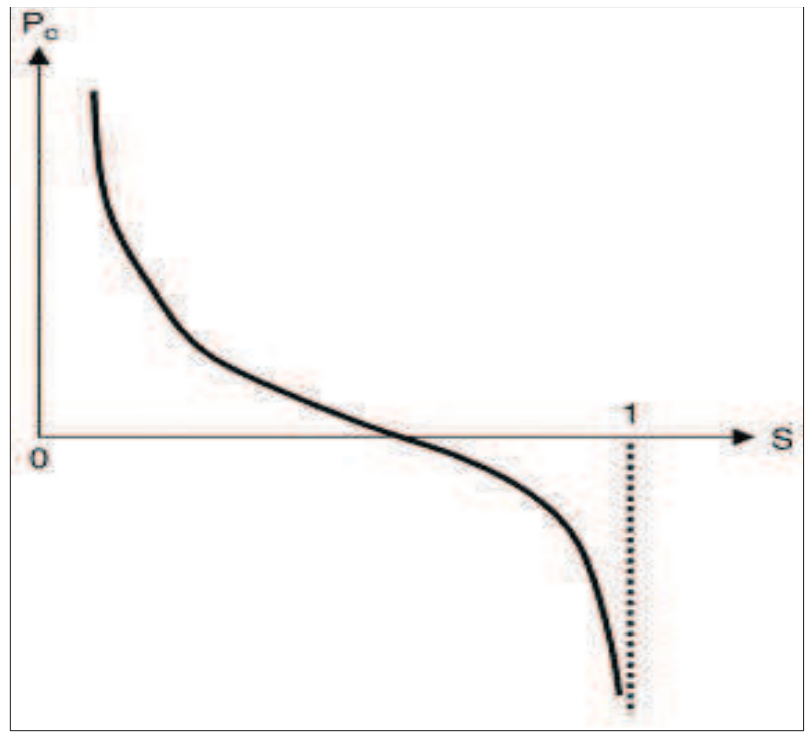

Fig. 11. Capillary pressure as a function of normalized wetting phase saturation.

Also, there are Corey- and LET-correlations for gas-water and gas-oil systems similar to the oil-water system. Correlation of the imbibition capillary pressure data depends on the type of application. For example, for water-oil system, see for example, (Pooladi-Darvish \& Firoozabadi, 2000), the capillary pressure and the normalized wetting phase saturation are correlated as,

$$
p_{c}=-B \ln S
$$

where $B$ is the capillary pressure parameter, which is equivalent to $\gamma\left(\frac{\phi}{K}\right)^{\frac{1}{2}}$, in the general form of the capillary pressure, Eq. (49), thus, $B \equiv-\gamma\left(\frac{\phi}{K}\right)^{\frac{1}{2}}$ and $J(S) \equiv \ln S$. Note that $J(S)$ is a scalar non-negative function. Capillary pressure as a function of normalized wetting phase (e.g. water) saturation is shown in Fig. 11. Also, the well known (van Genuchten, 1980; Brooks \& Corey, 1964) capillary pressure formulae which can be written as,

$$
\begin{gathered}
p_{c}=p_{0}\left(S^{-1 / m}-1\right)^{1-m}, \quad 0<m<1 \\
p_{c}=p_{d} S^{-1 / \lambda}, \quad 0.2<\lambda<3
\end{gathered}
$$

where $p_{0}$ is characteristic capillary pressure and $p_{d}$ is called entry pressure.

The capillary pressure $p_{c}$ is defined as a difference between the non-wetting and wetting phase pressures,

$$
p_{c}=p_{n}-p_{w}
$$

the total velocity defined as, 


$$
u=u_{w}+u_{n}
$$

the total mobility is given by,

$$
m(S)=m_{w}(S)+m_{n}(S)
$$

the fractional flow functions are,

$$
f_{w}(S)=\frac{m_{w}(S)}{m(S)}, \quad f_{n}(S)=\frac{m_{n}(S)}{m(S)}
$$

and the density difference is,

$$
\Delta \rho=\rho_{n}-\rho_{w}
$$

On the other hand, the molar density of wetting and nonwetting phases is given by,

$$
c_{w}=\sum_{i=1}^{N} c_{w i}, \quad c_{n}=\sum_{i=1}^{N} c_{n i}
$$

where $c_{w i}$ and $c_{n i}$ are the molar densities of component $i$ in the wetting phase and nonwetting phase phases, respectively. Therefore, the mole fraction of component $i$ in the respective phase is given as,

$$
x_{w i}=\frac{c_{w i}}{c_{w}}, \quad x_{n i}=\frac{c_{n i}}{c_{n}}, \quad i=1, \cdots, N
$$

The mole fraction balance implies that,

$$
\sum_{i=1}^{N} x_{w i}=1, \quad \sum_{i=1}^{N} x_{n i}=1
$$

Also, for the total mole fraction of $i^{\text {th }}$ component,

$$
\sum_{i=1}^{N} z_{i}=1
$$

Alternatively, Eq. (52) can be rewritten in the following form,

$$
\frac{\partial}{\partial t}\left[\phi\left(c_{w} x_{w i} S_{w}+c_{n} x_{n i} S_{n}\right)\right]+\nabla \cdot\left(c_{w} x_{w i} u_{w}+c_{n} x_{n i} u_{n}\right)=F_{i}, \quad i=1, \cdots, N
$$

$F_{i}$ may be written as,

$$
F_{i}=x_{w i} q_{w}+x_{n i} q_{n}, \quad i=1, \cdots, N
$$

where $q_{w}$ and $q_{n}$ are wetting phase and nonwetting phase phase flow rate, respectively. From Eqs. (32) and (72), one may deduce,

$$
c z_{i}=c_{w} x_{w i} S_{w}+c_{n} x_{n i} S_{n}=c_{w i} S_{w}+c_{n i} S_{n}, \quad i=1, \cdots, N
$$

If one uses the total mass variable $X$ of the system (Nolen, 1973; Young \& Stephenson, 1983),

$$
X=c_{w} S_{w}+c_{n} S_{n}
$$


Therefore,

$$
1=\frac{c_{w} S_{w}}{X}+\frac{c_{w} S_{n}}{X}=C_{w}+C_{n}
$$

where $C_{w}$ and $C_{n}$ are mass fractions of wetting- and nonwetting-phase of the system, respectively. It is noted that,

$$
C_{w}=1-C_{n}
$$

The total mole fraction of $i^{\text {th }}$ component, $z_{i}$, in terms of one phase (wetting phase) mass fraction and the wetting- and nonwetting-phase molar fractions, is given by,

$$
z_{i}=C_{w} x_{w i}+\left(1-C_{w}\right) x_{n i}, \quad i=1, \cdots, N
$$

The pressure equation can be obtained, using the concept of volume-balance, as follows,

$$
\phi C_{f} \frac{\partial p}{\partial t}+\sum_{i=1}^{N} \bar{V}_{i} \nabla \cdot\left(c_{w} x_{w i} u_{w}+c_{n} x_{n i} u_{n}\right)=\sum_{i=1}^{N} \bar{V}_{i} F_{i}, \quad i=1, \cdots, N
$$

where $C_{f}$ is the total fluid compressibility and $V_{i}$ is the total partial molar volume of the $i^{\text {th }}$ component. The distribution of the each component inside the two phases is restricted to the stable thermodynamic equilibrium in terms of phases' fugacities, $f_{w i}$ and $f_{n i}$ of the $i^{\text {th }}$ component. The stable thermodynamic equilibrium is given by minimizing the Gibbs free energy of the system Bear (1972); Chen (2007),

$$
f_{w i}\left(p_{w}, x_{w 1}, x_{w 2}, \cdots, x_{w N}\right)=f_{n i}\left(p_{n}, x_{n 1}, x_{n 2}, \cdots, x_{n N}\right), \quad i=1, \cdots, N
$$

The fugacity of the $i^{\text {th }}$ component is defined by,

$$
f_{\alpha i}=p_{\alpha} x_{o i} \phi_{\alpha i}, \quad \alpha=w, n, \quad i=1, \cdots, N
$$

$\phi_{\alpha i}, \alpha=w, n$ is the fugacity coefficient of the $i^{t h}$ component which will be defined below. The phase and volumetric behaviors, including the calculations of the fugacities, are modeled using the Peng-Robinson equation of state (Peng \& Robinson, 1976). Introducing the pressure of the phase, $p$ /alpha, which is given by Peng-Robinson two-parameter equation of state as,

$$
\begin{gathered}
p_{\alpha}=\frac{R T}{V_{\alpha}-b_{\alpha}}-\frac{a_{\alpha}(T)}{V_{\alpha}\left(V_{\alpha}+b_{\alpha}\right)+b_{\alpha}\left(V_{\alpha}-b_{\alpha}\right)}, \quad \alpha=w, n \\
a_{\alpha}=\sum_{i=1}^{N} \sum_{j=1}^{N} x_{i \alpha} x_{j \alpha}\left(1-\kappa_{i j}\right) \sqrt{a_{i} a_{j}}, \quad b_{\alpha}=\sum_{j=1}^{N} x_{i \alpha} b_{i}, \quad \alpha=w, n
\end{gathered}
$$

where $R$ is the universal gas of constant, $T$ is the temperature, $V_{\alpha}$ is the molar volume of the phase $\alpha, \kappa_{i j}$ is a binary interaction parameter between the components $i$ and $j, a_{i}$ and $a_{i}$ are empirical factor for the pure component $i$ given by,

$$
a_{i}=\Pi_{i a} \alpha_{i} \frac{R^{2} T_{i c}^{2}}{p_{i c}}, \quad b_{i}=\Pi_{i b} \frac{R T_{i c}}{p_{i c}}, \quad i=1, \cdots, N
$$

$T_{i c}$ and $p_{i c}$ are the critical temperature and pressure, 


$$
\begin{gathered}
\Pi_{i a}=0.45724, \quad \Pi_{i b}=0.077796, \quad \alpha_{i}=\left(1-\lambda_{i}\left[1-\sqrt{\frac{T}{T_{i c}}}\right]\right)^{2}, \\
\lambda_{i}=0.37464+1.5432 \omega_{i}-0.26992 \omega_{i}^{2}, \quad i=1, \cdots, N
\end{gathered}
$$

Eq. (72) can be rewritten in the following cubic form,

$$
Z_{\alpha}^{3}-\left(1-B_{\alpha}\right) Z_{\alpha}^{2}+\left(A_{\alpha}-2 B_{\alpha}-3 B_{\alpha}^{2}\right) Z_{\alpha}-\left(A_{\alpha} B_{\alpha}-B_{\alpha}^{2}-B_{\alpha}^{2}\right)=0, \quad \alpha=w, n
$$

where $Z_{\alpha}$ is the compressibility factor given by,

$$
Z_{\alpha}=\frac{b_{\alpha} V_{\alpha}}{R T}, \quad \alpha=w, n
$$

(Chen, 2007) explained how to solve the cubic algebraic equation, Eq. (64). The fugacity coefficient $\phi_{\alpha i}$ of the $i^{\text {th }}$ component is defined in terms of the compressibility factor $Z_{\alpha}$ as,

$$
\begin{aligned}
\ln \phi_{\alpha i}= & \frac{b_{i}}{b_{\alpha}}\left(Z_{\alpha}-1\right)-\ln \left(Z_{\alpha}-B_{\alpha}\right)-\frac{A_{\alpha}}{2 \sqrt{2} B_{\alpha}}\left(\frac{2}{a_{\alpha}} \sum_{j=1}^{N} x_{j \alpha}\left(1-\kappa_{i j}\right) \sqrt{a_{i} a_{j}}-\frac{b_{i}}{b_{\alpha}}\right) \\
& \cdot \ln \left(\frac{Z_{\alpha}+(1+\sqrt{2}) B_{\alpha}}{Z_{\alpha}-(1-\sqrt{2}) B_{\alpha}}\right)
\end{aligned}
$$

Deriving of this equation can be found in details in (Chen, 2007).

Using Eqs. (51)- (53) and (62)- (65) with some mathematical manipulation one can find,

$$
\frac{\partial\left(\phi \rho_{w} S_{w}\right)}{\partial t}=-\nabla \cdot \rho_{w} f_{w}(S)\left\{K m_{n}(S)\left(\frac{d p_{c}}{d S_{w}} \nabla S_{w}-\Delta \rho g \nabla z\right)+u\right\}+q_{w}
$$

Alternatively, in terms of pressure the flow equations may be rewritten in the form,

$$
\begin{gathered}
\frac{\partial\left(\phi \rho_{w} S_{w}\right)}{d p_{c}}\left(\frac{\partial p_{n}}{\partial t}-\frac{\partial p_{w}}{\partial t}\right)=\nabla \cdot \rho_{w}\left\{\frac{K k_{r w}}{\mu_{w}}\left(\nabla p_{w}-\rho_{w} g \nabla z\right)\right\}+q_{w} \\
\frac{\partial\left(\phi \rho_{n}\left(1-S_{w}\right)\right)}{d p_{c}}\left(\frac{\partial p_{n}}{\partial t}-\frac{\partial p_{w}}{\partial t}\right)=\nabla \cdot \rho_{n}\left\{\frac{K k_{r n}}{\mu_{n}}\left(\nabla p_{n}-\rho_{n} g \nabla z\right)\right\}+q_{n}
\end{gathered}
$$

Both models, Eq. (88) and Eqs. (89)- (90) are used intensively especially in the field of oil reservoir simulations.

\subsection{Three-phase compositional flow}

In three-phase compositional flow the governing equations will not has a big difference from the two-phase case. In this section we introduce the main points which distinguish the three-phase flow. On the other hand, we consider the black oil model as an example of the three-phase compositional flow instead of considering the general case to investigate such kind of complex flow. The black oil model is water-oil-gas system such that water represents the aqueous phase and oil represents oleic phase. The hydrocarbon in a reservoir is almost consists of oil and gas. Water is being naturally in the reservoir or injected in the secondary stage of oil recovery. Also, gas may be found naturally or/and injected as $\mathrm{CO}_{2}$ injection for the enhanced oil recovery stage. The governing equations may be extended to the three-phase flow. The generalized Darcy's law with mass transfer equations will remain the same as in Eqs. (30) and (31) with considering $\alpha=w, o, g$, thus each phase is represented by two equations, continuity and momentum. The index $\alpha$ denotes to the water $(w)$, oil $(o)$ and gas $(g)$, respectively. The solute transport equations is modified to suite the three-phase compositional flow as follow, 


$$
\frac{\partial\left(\phi c z_{i}\right)}{\partial t}+\nabla \cdot\left(c_{w} x_{w i} u_{w}+c_{o} x_{o i} u_{o}+c_{g} x_{g i} u_{g}\right)=F_{i}, \quad i=1, \cdots, N
$$

or

$$
\begin{aligned}
& \frac{\partial}{\partial t}\left[\phi\left(c_{w} x_{w i} S_{w}+c_{o} x_{o i} S_{o}+c_{g} x_{g i} S_{g}\right)\right]+\nabla \cdot\left(c_{w} x_{w i} u_{w}+c_{o} x_{o i} u_{o}+c_{g} x_{g i} u_{g}\right)= \\
& x_{w i} q_{w}+x_{o i} q_{o}+x_{g i} q_{g}, \quad i=1, \cdots, N
\end{aligned}
$$

Following (Stone, 1970; 1973) we assume that the water-oil and oil-gas relative permeabilities are given as the two-phase case,

$$
\begin{gathered}
k_{r w}\left(S_{w}\right)=k_{r w}^{0}\left(\frac{S_{w}-S_{w c}}{1-S_{w c}-S_{o r w}}\right)^{n_{w}} \\
k_{r g}\left(S_{g}\right)=k_{r g}^{0}\left(\frac{S_{g}-S_{g r}}{1-S_{w c}-S_{\text {org }}-S_{g r}}\right)^{n_{g}}
\end{gathered}
$$

where $S_{w c}$ is the connate water saturation, $S_{\text {org }}$ is the residual oil saturation to gas, $S_{\text {orw }}$ is the residual oil saturation to water, $S_{g r}$ is the residual gas saturation to water. $S_{w}=1-S_{\text {orw }}$. The intermediate-wetting phase (oil phase) relative permeabilities are given by,

$$
\begin{gathered}
k_{\text {row }}\left(S_{w}\right)=k_{\text {row }}^{0}\left(\frac{1-S_{w}-S_{\text {orw }}}{1-S_{w c}-S_{\text {orw }}}\right)^{n_{\text {ow }}} \\
k_{\text {rog }}\left(S_{g}\right)=k_{\text {rog }}^{0}\left(\frac{1-S_{w c}-S_{\text {org }}-S_{g}}{1-S_{w c}-S_{\text {org }}-S_{g r}}\right)^{n_{o g}}
\end{gathered}
$$

The intermediate-wetting phase relative permeability is given by,

$$
k_{r o}\left(S_{w}, S_{g}\right)=\frac{k_{\text {row }} k_{\text {rog }}}{k_{\text {norm }}}
$$

$k_{\text {norm }}$ may be setting as one or given by another formula as in the literature which will not mention here for breif.

\subsection{Numerical methods for multi-phase flow}

Much progress in the last three decades in numerical simulation of multi-phase flow with compositional and chemical effect. Both first-order finite difference and finite volume methods are used. First-order finite difference schemes has numerical dispersion issue, while the first-order finite volume has powerful features when used for two-phase flow simulation (Leveque, 2002). However, the later one has some limitations when applied to fractured media (Monteagudo \& Firoozabadi, 2007). Also, higher-order methods have less numerical dispersion and more accurate flow field calculations than the first-order methods. The combined mixed-hybrid finite element (MHFE) and discontinuous Galerkin (DG) methods have been used to simulate two-phase flow by (Hoteit \& Firoozabadi, 2005; 2006; Mikyska \& Firoozabadi, 2010). In the combined MHFE-DG methods, MHFE is used to solve the pressure equation with total velocity, and DG method is used to solve explicitly the species transport equations. Therefore, the parts are coupled using scheme such as the iterative IMplicit Pressure and Explicit Concentration (IMPEC) scheme. Also, (Sun et al., 2002) have used combined MHFE-DG methods to miscible displacement problems in porous media. 
The DG method (Wheeler, 1987; Sun \& Wheeler, 2005a;b; 2006) is derived from variational principles by integration over local cells, thus it is locally mass conservative by construction. In addition, the DG method has low numerical diffusion because higher-order approximations are used within cells and the cells interfaces are weakly enforced through the bilinear form. DG method is efficiently implementable on unstructured and nonconforming meshes.

The MHFE methods are based on a variational principle expressing an equilibrium or saddle point condition that can be satisfied locally on each element (Brezzi \& Fortin, 1991). It has an indefinite linear system of equations for pressure (scalar) and the total velocity (vector) but they definitized by appending as extra degrees of freedom the average pressures at the element edges.

\section{References}

Aissa, W. A. \& Mohammadein, A. A. (2006). Chemical reaction effects on combined forced and free convection flow of water at $4 \mathrm{c}$ past a semi-infinite vertical plate, J. Eng. Sci. Assiut Univ. 34: 1225-1237.

Bear, J. (1972). Dynamics of Fluids in Porous Media, Elsevier, New York.

Brezzi, F. \& Fortin, M. (1991). Mixed and hybrid finite element methods, Springer-Verlag, New York.

Brooks, R. H. \& Corey, A. T. (1964). Hydraulic properties of porous media, Hydrology Papers 3.

Chen, Z. (2007). Reservoir simulation: mathematical techniques in oil recovery, SIAM, USA.

Cheng, P. (1978). Heat transfer in geothermal systems, Advances in Heat Transfer 14: 1-105.

Cheng, P. (1981). Thermal dispersion effects on non-darcy convection flows in a saturated porous medium, Lett. Heat Mass Transfer 8: 267-270.

Dhir, V. K. (1994). Boiling and two-phase flow in porous media, Annu. Rev. Heat Transfer 5: $303-350$.

Doughty, C. \& Pruess, K. (1988). A semianalytical solution for heat-pipe effects near high-level nuclear waste packages buried in partially saturated geological media, Int. J. Heat Mass Transfer 31: 79-90.

El-Amin, M. F. (2004). Double dispersion effects on natural convection heat and mass transfer in non-darcy porous medium, Appl. Math. Comp. 156: 1-17.

El-Amin, M. F., Aissa, W. A. \& Salama, A. (2008). Effects of chemical reaction and double dispersion on non-darcy free convection heat and mass transfer, Transport in Porous Media 75: 93-109.

Elbashbeshy, E. M. A. (1997). Heat and mass transfer along a vertical plate with variable surface tension and concentration in the presence of magnetic field, Int. J. Eng. Sci. Math. Sci. 4: 515-522.

Hassanizadeh, S. M. \& Gray, W. (1979a). General conservation equations for multi-phase systems 2. mass, momenta, energy and entropy equations, Advances in Water Resources 2: 191-208.

Hassanizadeh, S. M. \& Gray, W. (1980). General conservation equations for multi-phase systems 3. constitutive theory for porous media, Advances in Water Resources 3: 25-40.

Hassanizadeh, S. M. \& Gray, W. G. (1979b). General conservation equations for multi-phase systems 1. averaging procedure, Advances in Water Resources 2: 131-144.

Herwig, H. \& Koch, M. (1991). Natural convection momentum and heat transfer in saturated highly porous mediaan asymptotic approach, Heat Mass Transfer 26: 169-174.

Hong, J. T. \& Tien, C. L. (1987). Analysis of thermal dispersion effect on vertical plate natural convection in porous media, Int. J. Heat Mass Transfer 30: 143-150. 
Hoteit, H. \& Firoozabadi, A. (2005). Multicomponent fluid flow by discontinuous galerkin mand mixed methods in unfractured and fractured media, Water Resour. Res. 41: W11412.

Hoteit, H. \& Firoozabadi, A. (2006). Compositional modeling by the combined discontinuous galerkin mand mixed methods, SPE Journal .

Lai, F. C. \& Kulacki, F. A. (1989). Thermal dispersion effect on non-darcy convection from horizontal surface in saturated porous media, Int. J. Heat Mass Transfer 32: 971-976.

Leal, L. G. (2007). Advanced transport phenomena, Cambridge University Press.

Leveque, R. J. (2002). Finite volume methods for hyperbolic problems, Cambridge Texts in Applied Mathematics, Cambridge University Press.

Mikyska, J. \& Firoozabadi, A. (2010). Implementation of higher-order methods for robust and efficient compositional simulation, J. Comput. Physics 229: 2898-2913.

Monteagudo, J. E. P. \& Firoozabadi, A. (2007). Control-volume model for simulation of water injection in fractured media: incorporating matrix heterogeneity and reservoir wettability effects, SPE Journal .

Mulolani, I. \& Rahman, M. (2000). Similarity analysis for natural convection from a vertical plate with distributed wall concentration, Int. J. Math. Math. Sci. 23: 319-334.

Murthy, P. V. S. N. \& Singh, P. (1997). Thermal dispersion effects on non-darcy natural convection with lateral mass flux, Heat Mass Transfer 33: 1-5.

Nolen, J. S. (1973). Numerical simulation of compositional phenomena in petroleum reservoirs, Reprint Series, SPE, Dallas, 11, 268284.

Peng, D.-Y. \& Robinson, D. B. (1976). A new two-constant equation of state, Industrial and Engineering Chemistry Fundamentals 15: 59-64.

Plumb, O. A. (1983). The effect of thermal dispersion on heat transfer in packed bed boundary layers, In: Proc. 1st ASME/JSME Thermal Engng. Joint Conf., Vol. 2, pp. 17-21.

Pooladi-Darvish, M. \& Firoozabadi, A. (2000). Co-current and counter-current imbibition in a water-wet matrix block, SPE Journal 5: 3-11.

Prasad, K. V., Abel, S. \& Datti, P. S. (2003). Diffusion of chemically reactive species of a non-newtonian fluid immersed in a porous medium over a stretching sheet, Int. J. Non-Linear Mech. 38: 651-657.

Salama, A. \& van Geel, P. J. (2008a). Flow and solute transport in saturated porous media: 1 the continuum hypothesis, J Porous Media 11: 403-413.

Salama, A. \& van Geel, P. J. (2008b). Flow and solute transport in saturated porous media: 2 violating the continuum hypothesis, J Porous Media 11: 421-441.

Starikovicius, V. (2003). The multiphase fl ow and heat transfer in porous media, Berichte des Fraunhofer ITWM 55: 1-30.

Stone, H. (1970). Probability model for estimating three-phase relative permeability, JPT 214.

Stone, H. (1973). Estimation of three-phase relative permeability and residual oil data, J. Cdn. Pet. Tech. 13: 53.

Sun, S., Riviere, B. \& Wheeler, M. F. (2002). A combined mixed finite element and discontinuous galerkin method for miscible displacement problems in porous media, Proceedings of International Symposium on Computational and Applied PDEs, Zhangjiajie National Park of China, 321-341.

Sun, S. \& Wheeler, M. F. (2005a). Discontinuous galerkin methods for coupled flow and reactive transport problems, Appl. Num. Math. 52: 273-298.

Sun, S. \& Wheeler, M. F. (2005b). Symmetric and nonsymetric discontinuous galerkin methods for reactive transport in porous media, SIAM J. Numer. Anal. 43: 195-219. 
Sun, S. \& Wheeler, M. F. (2006). Analysis of discontinuous galerkin methods for multi-components reactive transport problem, Comput. Math. Appl. 52: 637-650.

Telles, R. S. \& V.Trevisan, O. (1993). Dispersion in heat and mass transfer natural convection along vertical boundaries in porous media, Int. J. Heat Mass Transfer 36: 1357-1365.

Udell, K. S. (1985). Heat transfer in porous media considering phase change and capillarity the heat pipe effect, Int. J. Heat Mass Transfer 28: 485-495.

van Genuchten, M. (1980). A closed-form equation for predicting the hydraulic conductivity of unsaturated soils, Soil Sci. Soc. Am. J. 44: 892-898.

Wheeler, M. F. (1987). An elliptic collocation finite element method with interior penalties, SIAM J. Numer. Anal. 15: 152-161.

Whitaker, S. (1967). Diffusion and dispersion in porous media, AIChE 13: 420-427.

Whitaker, S. (1977). Simultaneous heta, mass, and momentum transfer in porous media: A theory of drying, Advances in Heat Transfer 13: 119-203.

Young, L. C. \& Stephenson, R. E. (1983). A generalized compositional approach for reservoir simulation, SPE Journal 23: 727-742. 


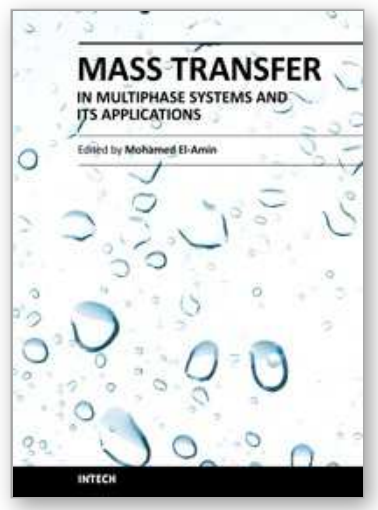

\author{
Mass Transfer in Multiphase Systems and its Applications \\ Edited by Prof. Mohamed El-Amin
}

ISBN 978-953-307-215-9

Hard cover, 780 pages

Publisher InTech

Published online 11, February, 2011

Published in print edition February, 2011

This book covers a number of developing topics in mass transfer processes in multiphase systems for a variety of applications. The book effectively blends theoretical, numerical, modeling and experimental aspects of mass transfer in multiphase systems that are usually encountered in many research areas such as chemical, reactor, environmental and petroleum engineering. From biological and chemical reactors to paper and wood industry and all the way to thin film, the 31 chapters of this book serve as an important reference for any researcher or engineer working in the field of mass transfer and related topics.

\title{
How to reference
}

In order to correctly reference this scholarly work, feel free to copy and paste the following:

M.F. El-Amin, Amgad Salama and Shuyu Sun (2011). Solute Transport With Chemical Reaction in Singleand Multi-Phase Flow in Porous Media, Mass Transfer in Multiphase Systems and its Applications, Prof. Mohamed El-Amin (Ed.), ISBN: 978-953-307-215-9, InTech, Available from: http://www.intechopen.com/books/masstransfer-in-multiphase-systems-and-its-applications/solute-transport-with-chemical-reaction-in-singleand-multiphase-flow-in-porous-media

\section{INTECH}

open science | open minds

\section{InTech Europe}

University Campus STeP Ri Slavka Krautzeka 83/A 51000 Rijeka, Croatia Phone: +385 (51) 770447

Fax: +385 (51) 686166 www.intechopen.com

\section{InTech China}

Unit 405, Office Block, Hotel Equatorial Shanghai No.65, Yan An Road (West), Shanghai, 200040, China 中国上海市延安西路65号上海国际贵都大饭店办公楼405单元 Phone: +86-21-62489820

Fax: +86-21-62489821 
(C) 2011 The Author(s). Licensee IntechOpen. This chapter is distributed under the terms of the Creative Commons Attribution-NonCommercialShareAlike-3.0 License, which permits use, distribution and reproduction for non-commercial purposes, provided the original is properly cited and derivative works building on this content are distributed under the same license. 